DOI: https://doi.org/10.14311/TPFM.2022.017

\title{
HYBRID FICTITIOUS DOMAIN-IMMERSED BOUNDARY METHOD IN CFD-BASED TOPOLOGY OPTIMIZATION
}

\author{
Lucie Kubíćková ${ }^{1,2}$, Martin Isoz ${ }^{1,2}$ \\ ${ }^{1}$ Department of Mathematics, Faculty of Chemical Engineering, University of Chemistry and \\ Technology, Technická 5, Prague 166 28, Czech Republic \\ ${ }^{2}$ Czech Academy of Sciences, Institute of Thermomechanics, Dolejśkova 5, Prague 18200 , \\ Czech Republic
}

\begin{abstract}
Advances in technological development, especially in 3D printing, allow engineers to design components with almost arbitrary shape and connectivity. Consequently, more and more attention is being directed towards a highly-specialized application-driven component design based on topology optimization (TO). In the present work, we propose a methodology enabling TO of components in contact with flowing fluids. In particular, the optimization itself is based on multi-objective evolutionary algorithms (MOEAs) with the component geometry encoded using a binary representation. The optimization criteria are evaluated via computational fluid dynamics (CFD). The main novelty of the proposed TO framework lies in its robustness and effectiveness achieved by utilizing a single computational mesh for all the tested designs and projecting the specific components shapes onto it by the means of an immersed boundary method. The new methodology capabilities are illustrated on a shape optimization of a diffuser equipped as a part of an ejector. The optimization goal was to increase the ejector energy efficiency. The newly proposed methodology was able to identify a design by roughly $9 \%$ more efficient than an alternative one found utilizing a previously published and less general optimization approach.
\end{abstract}

Keywords: HFDIB, topology optimization, CFD, OpenFOAM.

\section{Introduction}

Thanks to the ongoing technological advance, engineers can design and manufacture device components with almost arbitrary shape and connectivity. Thus, the component topology can be designed precisely to a specific application, which promotes usage and development of various topology optimization (TO) methods [1]. According to the literature, there are two approaches to the TO, deterministic and stochastic one [2]. In both the approaches, a representation of the optimized structure is required. Such a representation may be supplied for example by Bézier curves, Voronoï representation, or using a structure binary representation $[2,3]$. Next, both the approaches need a cost function, that can be optimized based on a specified optimization criteria. Nonetheless, there is a fundamental difference between the two approaches.

The deterministic approach uses standard methods of mathematical optimization (SMMO), e.g. gradient techniques or methods based on sensitivity analysis [2], to optimize the cost function. The deterministic approach was used for example by Jensen and Sigmund [4] to optimize a material distribution in a photonic crystal or by Ma et al. [5] to design a structure of acoustic cloaks. Nevertheless, the SMMO are, by definition, local. Thus, they start from a preset initial topology, test a small amount of intermediate topologies and converge to a local optimum. Thus, the deterministic approach is efficient for finding a local optimum of the cost function. However, to estimate the global optimum, it is necessary to either conduct an analysis of the problem specific cost function or to restart the TO multiple times using different starting topologies.

On the other hand, the stochastic approach can converge towards the global optimum in a single optimization run, since the stochastic methods are able to explore a significant part of the parametric space using random operators [6]. A popular group of the stochastic optimization methods are multiobjective evolutionary algorithms (MOEAs) [6]. Due to their robustness, MOEAs have been already used by numerous authors, e.g. by Hur et al. [7] to perform a topology optimization of mechanically stressed component or by Boichot and Fan [8] to solve area-to-point heat conduction problems.

In the present contribution, we focus on $\mathrm{TO}$ of device components that come in contact with flowing fluids. More specifically, we concentrate on the cases in which the cost function evaluation 
is based on methods of computational fluid dynamics (CFD). Optimization of such components was already presented by e.g. Koguchi et al. [9], Kubo et al. [10]. However, up to the authors knowledge, all the published CFD-leveraging TOs are performed via the deterministic approach and SMMO. In this work, we base the TO on the stochastic MOEAs combined with the binary representation of the component structure, which allows for development of a robust and general TO framework that requires no prior knowledge of the cost function properties nor of the expected optimized component topology.

The proposed TO methodology is based on a connection of MOEAs and CFD. The robustness and stochasticity of MOEAs demands numerous cost function evaluations. Thus, basing the cost function on CFD makes the final methodology computationally challenging. In order to make the optimization more effective, we used an approach similar to the one in $[9,10]$, i.e. we extended the standard CFD methods by an immersed boundary method, the hybrid fictitious domain-immersed boundary (HFDIB) method [11] in particular, which allowed us to use a single computational mesh for all the tested topologies and thus to lower the necessary computational efforts.

The methodology is illustrated on a proof-of-concept problem of shape optimization of a diffuser equipped as a part of an ejector. The optimization goal is to increase the ejector energy efficiency. This particular problem was chosen, because we have already conducted an optimization of the same component using a similar but less general optimization methodology, see [12]. The less general methodology was validated on experiments; thus, it gave us a referential solution, which was used to evaluate the proposed methodology capabilities.

\section{Methods}

The present section is devoted to a description of the proposed methodology. We start with a general description of the optimization problem and MOEAs. Next, the illustrative example of the diffuser shape optimization is used to present the optimization parameter space and cost function. Lastly, we discuss the overall optimization strategy.

\subsection{Optimization problem and multiobjective evolutionary algorithms}

Optimization problem Let $\mathcal{P} \subset \mathbb{R}^{n}$ be a parameter space, $\mathcal{O} \subset \mathbb{R}^{m}$ an objective space and $f_{\text {cost }}: \mathcal{P} \rightarrow \mathcal{O}$ mark a vector-valued cost function. Then a general multiobjective-multiparameter optimization problem can be written as

$$
\text { find } \tilde{\boldsymbol{p}} \in \mathcal{P} \text { such that } \min _{\boldsymbol{p} \in \mathcal{P}} \boldsymbol{f}_{\text {cost }}(\boldsymbol{p})=\tilde{\boldsymbol{o}}:=\boldsymbol{f}_{\text {cost }}(\tilde{\boldsymbol{p}}) \text {, }
$$

where, in the studied setting, $\boldsymbol{p}$ is a vector of parameters defining the component topology and the vector $\boldsymbol{o}$ contains optimization criteria supplied by a CFD-based cost function.

Multiobjective evolutionary algorithms The problem (1) can be solved using multiobjective evolutionary algorithms (MOEAs). In general, MOEAs mimic principles of natural evolution using random operators. In particular, MOEAs work with a population of individua, where one individuum is defined by a vector of parameters $\boldsymbol{p}$ and a criteria vector $\boldsymbol{o}$. Then, the algorithms work in cycles corresponding to generation and randomly recombines the properties of the best performing individua of each generation in order to identify the fittest individua overall, i.e. the Pareto optimal set of the problem (1). The specific MOEA chosen in this work was the NSGA-II algorithm of Deb et al. [6].

\subsection{Problem parameter space and cost function}

Independently of the chosen solution algorithm, the problem (1) needs to be supplied by a parameter space $\mathcal{P}$ and objective space $\mathcal{O}$. Without loss of generality, we describe the structure of both the spaces using the proof-of-concept problem of the diffuser shape optimization. The diffuser equipped as part of an ejector is illustrated in Fig. 1-a). The parameter space $\mathcal{P}$ comprises all the possible component topologies. However, the size of $\mathcal{P}$ is limited by defining the component maximum dimensions, see Fig. 1-c). Next, new component (diffuser) topologies are created by filling the biggest acceptable shape with material. To work with a finite number of topologies, the parameter space is discretized using a binary representation of the biggest acceptable shape. 


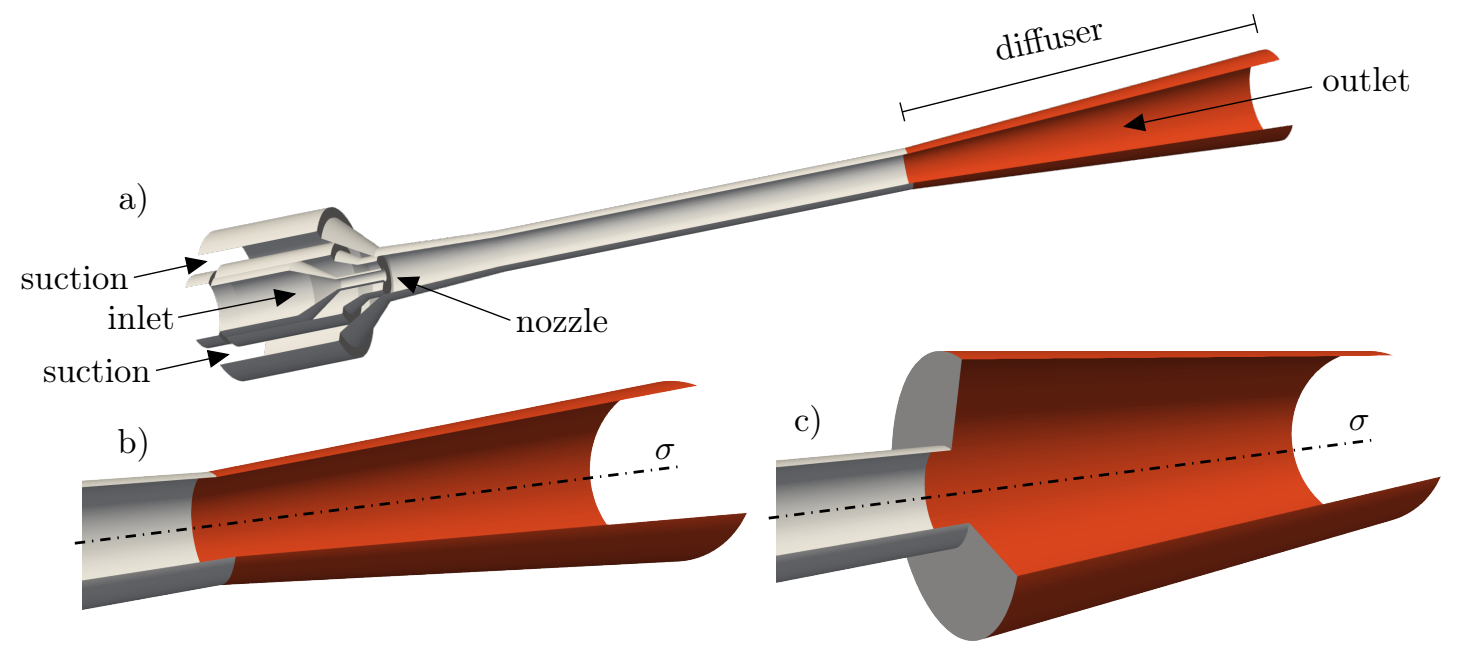

Figure 1: (a) Ejector with diffuser part highlighted in red. (b) Original diffuser shape with indicated symmetry axis $\sigma$. (c) The biggest acceptable diffuser shape.

Binary representation The formation of the binary representation for the diffuser, which is axisymmetric, is given in Fig. 2. The formation starts from the biggest acceptable shape, see Fig. 2-a), which was discretized into a finite number of topology cells, see Fig. 2-b). Then, each cell is filled with either material or void, see Fig.2-c). Afterwards, the cells with material are assigned ones and cells with void zeros, and the topology is transcribed into a binary representation matrix $B_{p}$, see Fig. 2-d). Lastly, the matrix $B_{\boldsymbol{p}}$ is reshaped into the parametric vector $\boldsymbol{p}$.

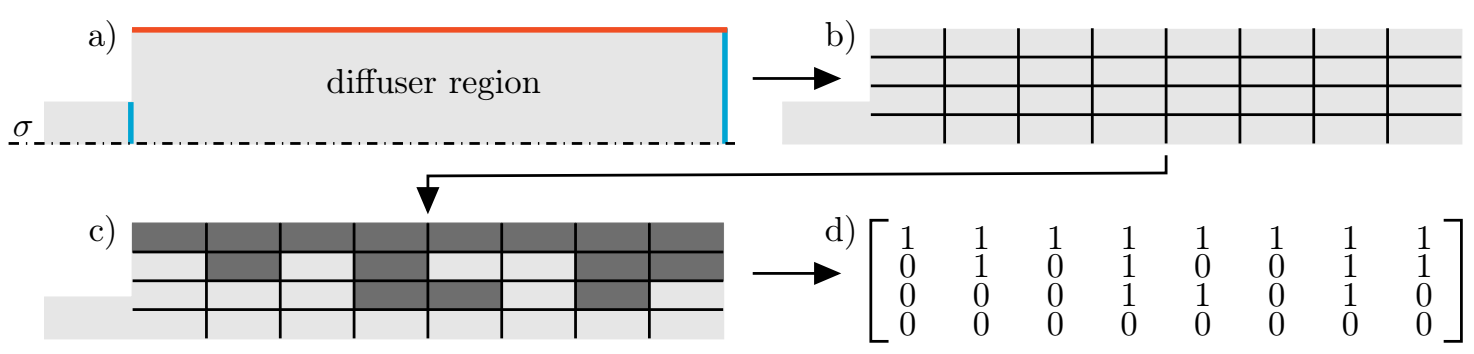

Figure 2: Formation of the topology binary representation in a 2D axi-symmetric projection. (a) Diffuser region with indicated symmetry axis $\sigma$ and highlighted inlet and outlet (blue lines) and outer wall (red line). (b) Diffuser region discretized into topology cells. (c) Exemplary diffuser topology. Topology cells are filled with either material or void. (d) Transcription into the binary representation matrix.

Acceptable topologies Let us note that the stochastic approach to TO is based on a random recombination of the vectors $\boldsymbol{p}$. In particular, the optimization algorithm generates a vector $\boldsymbol{p}$ and passes it to the cost function, in which the vector $\boldsymbol{p}$ is reshaped to $B_{\boldsymbol{p}}$ and $B_{\boldsymbol{p}}$ is used for the CFD model construction. Thus, MOEA by itself does not understand the geometrical meaning of the vectors $\boldsymbol{p}$ and can generate $\boldsymbol{p}$ leading to physically unacceptable component geometries. In fact, the vast majority of $B_{\boldsymbol{p}}$ originating from randomly generated $\boldsymbol{p}$ leads to geometries for which a reasonable CFD model cannot be constructed. Consequently, the MOEA optimization with CFDbased cost function needs to be endowed with topology acceptability conditions, which are usually problem-specific.

For the particular case of the diffuser shape optimization, we defined three conditions the topology must satisfy to be acceptable, (i) the topology must be permeable, i.e. there must exist a continuous way between the inlet and outlet (see blue lines in Fig. 2-a), (ii) it must be manufacturable, so all the material must be connected to the outer wall (see the red line in Fig. 2a), and (iii) it must not contain closed cavities. The geometries containing closed cavities are unacceptable because they have the exact same flow geometry as shapes that have the cavities filled with material. Examples of acceptable and unacceptable topologies are shown in Fig. 3. 
a)
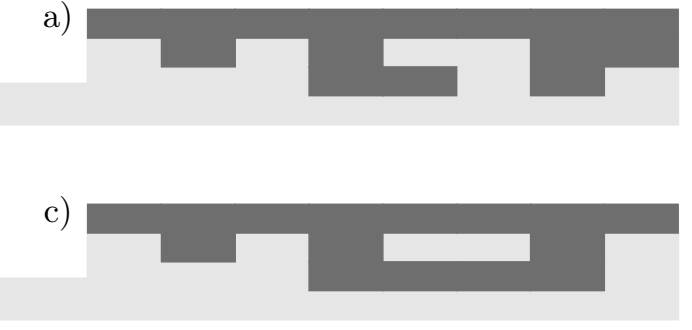

b)
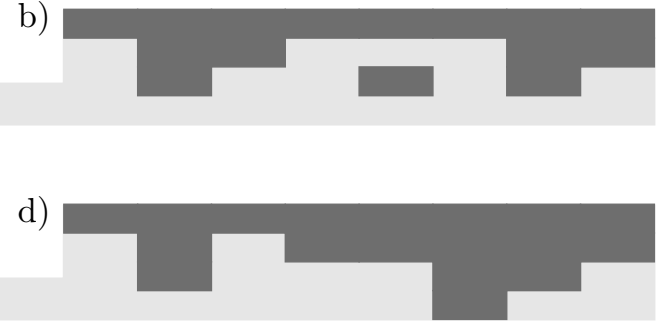

Figure 3: (a) Acceptable topology. (b) Topology violating the manufacturability condition. (c) Topology with a closed cavity inside the material. (d) Impermeable topology.

Optimization criteria Using the topology acceptability conditions, it is either possible to reduce the space $\mathcal{P}$ to the acceptable topologies $\mathcal{P}_{a}$, or to extend the CFD-based cost function in a way that the optimization algorithm can differentiate between acceptable and unacceptable component topologies, i.e. between $\boldsymbol{p} \in \mathcal{P}_{a}$ and $\boldsymbol{p} \in \mathcal{P} \backslash \mathcal{P}_{a}$. In the present work, we opted for the latter and defined the problem cost function as

$$
\boldsymbol{o}:=\boldsymbol{f}_{\text {cost }}(\boldsymbol{p})=\boldsymbol{Q}(\boldsymbol{p})+\boldsymbol{w} S(\boldsymbol{p}), \boldsymbol{Q}(\boldsymbol{p})=\left\{\begin{array}{cl}
\boldsymbol{q}(\boldsymbol{p}) & \text { if } \boldsymbol{p} \in \mathcal{P}_{a} \\
\mathbf{0} & \text { else }
\end{array}, S(\boldsymbol{p})=\left\{\begin{array}{cl}
0 & \text { if } \boldsymbol{p} \in \mathcal{P}_{a} \\
s(\boldsymbol{p}) & \text { else }
\end{array}\right.\right.
$$

where $\boldsymbol{q}$ are the optimization criteria computed using CFD, $s$ is a penalty function based on the problem-specific topology acceptability criteria, and $\boldsymbol{w}$ is a vector of weights. The penalty function used in the diffuser shape optimization was defined as $s(\boldsymbol{p})=\Gamma_{1} \gamma_{1}+\Gamma_{0} \gamma_{0}+\Gamma_{F} \gamma_{F}$, with $\gamma_{1}$ being the number of topology cells with material not connected to the outer wall, $\gamma_{0}$ the number of cells inside closed cavities and $\gamma_{F}$ the number of columns of $B_{p}$ not connected to the topology inlet, i.e. unreachable for the fluid. Finally, $\Gamma_{1}, \Gamma_{0}$ and $\Gamma_{F}$ are problem-specific method hyper-parameters. From the (2), it can be seen that $\mathcal{P}$ contains all the possible vectors $\boldsymbol{p}$. However, the optimization criteria for $\boldsymbol{p} \notin \mathcal{P}_{a}$ are computed using an algebraic relation and do not require the CFD model construction.

CFD model The optimization criteria for any $\boldsymbol{p} \in \mathcal{P}_{a}$ are evaluated using CFD. The construction and evaluation of the CFD models was done using standard CFD methods as implemented in the open-source finite volume (FV) $\mathrm{C}++\mathrm{CFD}$ library OpenFOAM [13]. However, in order to allow for usage of a single FV mesh for all the CFD computations, the CFD methodology was extended by a custom implementation of the immersed boundary method, HFDIB, presented in [11].

The flow affecting the optimized component is simulated via a standard set of the NavierStokes equations. For example, in the diffuser shape optimization, the fluid was considered to be Newtonian and the flow was assumed to be steady-state, incompressible and isothermal. Thus, the problem was governed by equations of the form

$$
\begin{array}{cc}
\mathcal{M}(\boldsymbol{u})=-\nabla \tilde{p}+\boldsymbol{g}+\boldsymbol{f}_{\mathrm{ib}}, & \mathcal{M}(\boldsymbol{u})=\nabla \cdot(\boldsymbol{u} \otimes \boldsymbol{u})-\nabla \boldsymbol{\tau} \\
\nabla \cdot \boldsymbol{u}=0 & \boldsymbol{f}_{\mathrm{ib}}=\operatorname{cell}(\lambda)\left(\mathcal{M}\left(\boldsymbol{u}_{\mathrm{ib}}\right)+\nabla \tilde{p}-\boldsymbol{g}\right)
\end{array}
$$

where $\boldsymbol{u}$ and $\tilde{p}$ are velocity and kinematic pressure, respectively, $\boldsymbol{g}$ is gravitational acceleration, $\boldsymbol{\tau}$ stands for viscous stress tensor and $\boldsymbol{f}_{\mathrm{ib}}$ is the force term generated by HFDIB.

Let us recall that geometrically, the FV mesh $\Omega$ on which the equations (3) are solved corresponds to the biggest acceptable shape. However, the binary representation $B_{\boldsymbol{p}}$ defined by $\boldsymbol{p} \in \mathcal{P}_{a}$ effectively enforces the division $\Omega=\Omega_{\mathrm{s}} \cup \Omega_{\mathrm{f}}, \Omega_{\mathrm{s}} \cap \Omega_{\mathrm{f}}=\emptyset$, where $\Omega_{\mathrm{s}}$ is the part of $\Omega$ filled with the material and $\Omega_{\mathrm{f}}$ is available for the fluid flow. The term $f_{\mathrm{ib}}$ in (3) represents the force that needs to act on the fluid to prevent the flow from entering $\Omega_{\mathrm{s}}$ and to enforce the prescribed boundary conditions on $\partial \Omega_{\mathrm{ib}}$. The term $\boldsymbol{f}_{\mathrm{ib}}$ depends on a scalar field $\lambda \in[0,1]$, which is a projection of $B_{\boldsymbol{p}}$ onto the FV mesh $\Omega$. The FV grid $\Omega$ is usually significantly more refined than the topological discretization encoded in $B_{\boldsymbol{p}}$, which allows for smoothing of the $\lambda$ field and removing the castellated component boundaries caused by the coarse topology discretization.

In the diffuser shape optimization, the mesh $\Omega$ was axi-symmetric and its boundary $\partial \Omega$ was divided as

$$
\partial \Omega=\partial \Omega_{\text {inlet }} \cup \partial \Omega_{\text {outlet }} \cup \partial \Omega_{\text {wall }} \cup \partial \Omega_{\text {ib }}
$$




\begin{tabular}{lclc}
\hline Boundary & Conditions & Boundary & Conditions \\
\hline$\partial \Omega_{\text {inlet }}$ & $\boldsymbol{u}=-Q_{\text {inlet }} \boldsymbol{n}_{\text {inlet }} / A_{\text {inlet }}$ & $\partial \Omega_{\text {wall }}$ & $\begin{array}{c}\boldsymbol{u}=(0,0,0)^{\mathrm{T}} \\
\boldsymbol{n}_{\text {wall }} \cdot \nabla p=0\end{array}$ \\
\hline $\boldsymbol{n}_{\text {inlet }} \cdot \nabla p=0$ & & $\boldsymbol{u}=(0,0,0)^{\mathrm{T}}$ \\
& $\boldsymbol{n}_{\text {outlet }} \cdot \nabla \boldsymbol{u}=(0,0,0)^{\mathrm{T}}$ & $\partial \Omega_{\mathrm{ib}}$ & $\boldsymbol{n}_{\text {ib }} \cdot \nabla p=0$ \\
\hline
\end{tabular}

Table 1: Prescribed boundary conditions, where $Q$ marks the fluid volumetric flow rate, $\boldsymbol{n}$ is the outer unit normal to the boundary and $A$ is area.

where $\partial \Omega_{\text {inlet }}$ and $\partial \Omega_{\text {outlet }}$ represent the fluid inlet and outlet, respectively. Next, $\partial \Omega_{\text {wall }}$ represents solid boundaries and $\partial \Omega_{\mathrm{ib}}$ the immersed boundary. The governing equations (3) were completed by boundary conditions for velocity $\boldsymbol{u}$ and kinematic pressure $\tilde{p}$ listed in Tab. 1 .

Finally, the CFD model was simulated in a laminar regime and the resulting velocity and pressure fields were used to estimate the diffuser energy efficiency. The energy efficiency was approximated by a so-called pressure recovery factor $c_{p}$ that indicates the pressure recovery efficiency of the diffuser and is strongly correlated to the overall ejector efficiency [12]. The pressure recovery factor was defined in agreement with [14] as

$$
c_{p}=\frac{1}{\frac{1}{2} \rho u_{\mathrm{ref}}^{2}}\left[\frac{1}{\left\|A_{\text {outlet }}\right\|} \int_{A_{\text {outlet }}} p d S-\frac{1}{\left\|A_{\text {ref }}\right\|} \int_{A_{\text {ref }}} p d S\right]=:-o(\boldsymbol{p})
$$

where $\rho$ is the fluid density, $p$ is the static pressure and $u_{\text {ref }}$ is the mean streamvise velocity over area $A_{\text {ref }}$. The areas $A_{\text {outlet }}$ and $A_{\text {ref }}$ were cross sectional areas perpendicular to the main flow direction placed at the outlet and at a reference position one centimeter before the diffuser, respectively. Note that this proof-of-concept optimization was single-objective as $\boldsymbol{o}=-c_{p}$. However, the methodology itself is general.

\subsection{Optimization strategy}

As it was stated above, the parametric space $\mathcal{P}$ consists of both acceptable and unacceptable topologies, i.e. $\mathcal{P}=\mathcal{P}_{a} \cup \mathcal{P}_{n}, \mathcal{P}_{n}=\mathcal{P} \backslash \mathcal{P}_{a}$ and the cost function distinguishes between $\mathcal{P}_{a}$ and $\mathcal{P}_{n}$ and uses either CFD or penalty function to compute optimization criteria. However, it holds that $\left|\mathcal{P}_{n}\right| \sim|\mathcal{P}|$ and consequently $\left|\mathcal{P}_{n}\right| \gg\left|\mathcal{P}_{a}\right|$. Thus, to obtain a robust optimization framework, a specific strategy is needed. The proposed strategy is illustrated in Fig. 4 and it comprises three steps, preliminary optimizations (POs), initial optimizations (IOs) and the final optimization (FO).

The preliminary optimizations purpose is to set the penalty function hyper-parameters, $\boldsymbol{\Gamma}$. The POs use a modified cost function, since the CFD results are not relevant at this time. The modified cost function is

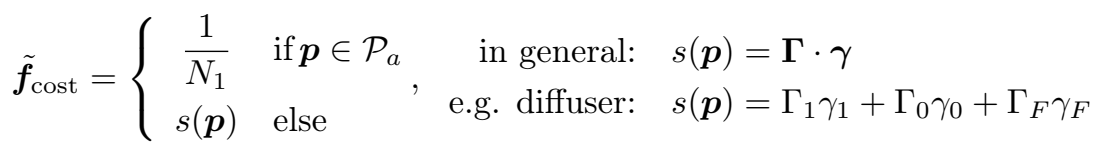

where $N_{1}$ is a total number of cells containing material. A single PO then starts from a randomly generated population and it is terminated, when the population is dominated by a single acceptable shape. The optimization convergence rate is strongly dependent on the hyper-parameter values. Hence, POs are used to tune the hyper-parameters $\boldsymbol{\Gamma}$ to make the MOEA convergence as fast as possible. In a general case, tuning $\Gamma$ requires to run low tens of POs.

Next, the initial optimizations are performed. The purpose of IOs is to create a representative sample of acceptable topologies, $\boldsymbol{P}_{A}=\left\{\boldsymbol{p}_{k} \in \mathcal{P}_{a}\right\}_{k=1}^{n}$. An IO uses the cost function (6) with the tuned hyper-parameters and starts from a randomly generated population. The first acceptable topology the IO finds quickly propagates in the population until the whole population is dominated by it. Then the dominant topology slowly changes towards a specific topology enforced by the definition of the cost function (6). Ergo, each IO always gives a single $\boldsymbol{p} \in \mathcal{P}_{a}$, provided it successfully converges. Hence to create a sample $\boldsymbol{P}_{A}$ of size $n$, it is necessary to run roughly $n$ IOs.

The last step of the proposed TO framework is the final optimization, the goal of which is to get the optimized component itself. The FO initial population is formed by the representative sample 


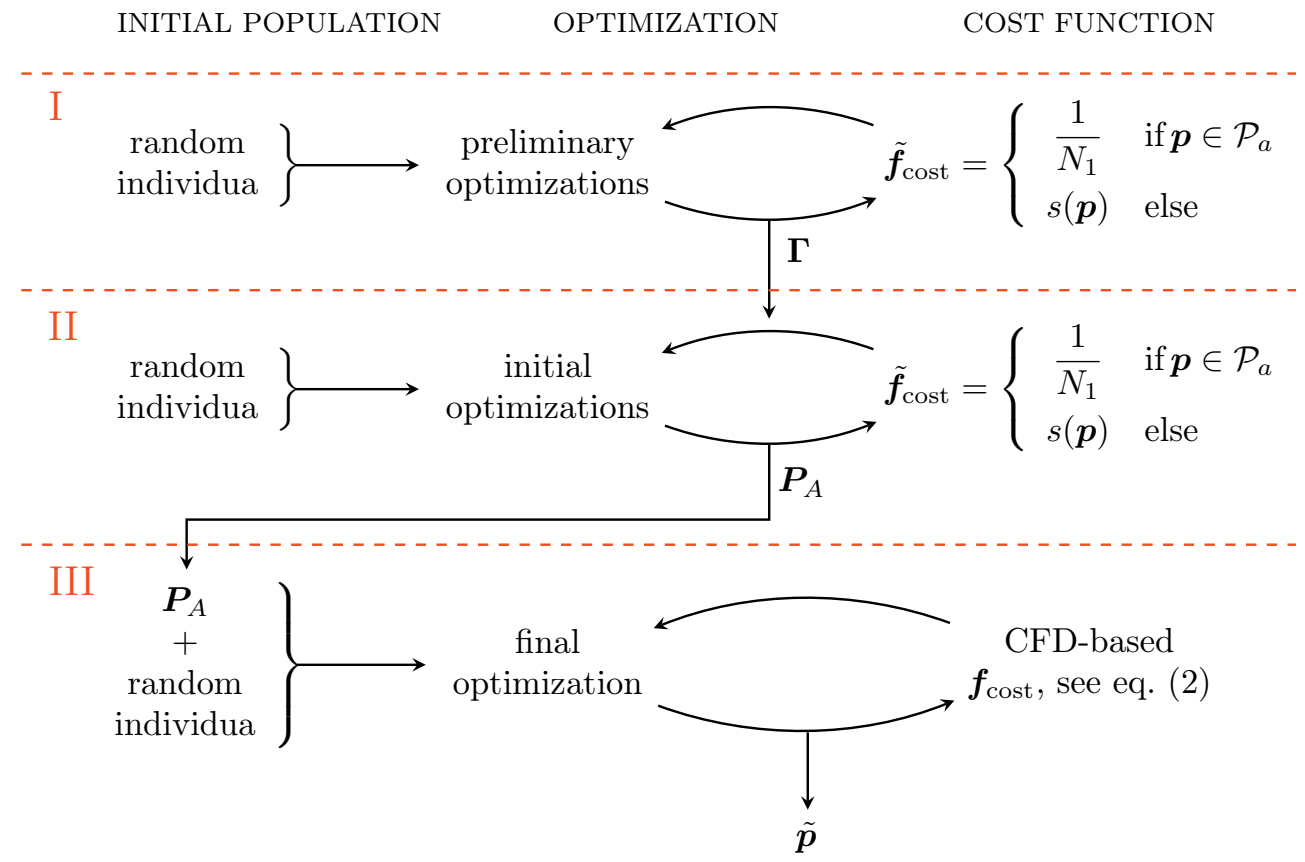

Figure 4: Schematics of the optimization strategy.

$\boldsymbol{P}_{A}$, which is extended by randomly generated topologies. During the framework design, it was found out that running the FO with a population comprising $\boldsymbol{P}_{A}$ of size $n$ extended by $n / 2$ random shapes significantly improves the NSGA-II convergence properties. In particular, this extension allows the algorithm to find acceptable topologies that are not similar to any $\boldsymbol{p} \in \boldsymbol{P}_{A}$. The FO uses the CFD-based form of the cost function (2) and provides an estimate of the problem (1) global solution.

\section{Results}

To solve the proof-of-concept optimization problem, we first needed to prepare the CFD model computational domain. The diffuser axi-symmetry was leveraged to prepare a $2 \mathrm{D}$ axisymmetric computational mesh $\Omega$ corresponding to the biggest acceptable diffuser shape, which was $13 \mathrm{~cm}$ long with the outlet diameter of $5 \mathrm{~cm}$. Based on a mesh resolution independence study, see Fig. 5a), c)-e $)^{1}$, we discretized $\Omega$ into $\mathrm{FV}$ cells of characteristic size $\simeq 0.5 \mathrm{~mm}$. As a result, the diffuser region consisted of $13000 \mathrm{FV}$ cells. Then, we discretized the diffuser region into 520 topology cells. Hence, one topology cell corresponded to $5 \times 5 \mathrm{FV}$ cells. Lastly, we set the $Q_{\text {inlet }}$ to $1.5 \mathrm{l} / \mathrm{s}$ and the fluid kinematic viscosity to $10^{-3} \mathrm{~m}^{2} / \mathrm{s}$, resulting in the Reynolds number $\mathrm{Re}=100$ at the diffuser inlet.

Next, we followed the described optimization strategy. We first ran 12 POs and set the hyperparameters $\Gamma_{1}, \Gamma_{0}$ and $\Gamma_{F}$ to 5.0, 10.0 and 300.0, respectively. With these hyper-parameter values and utilizing a standard laptop, the POs were able to converge in about 2 minutes. Then, 305 IOs were performed, 300 of which successfully converged to a unique acceptable shape. Hence, the obtained representative sample $\mathcal{P}_{A}$ had the size of 300 . This sample was mixed with 150 randomly generated topologies to obtain an initial population of 450 unique individua for the final optimization.

The FO ran for 70 generations. Thus, it evaluated $70 \cdot 450=31500$ topologies. The optimization ran for roughly 56 hours on a cluster with 90 CPUs using a parallelized population evaluation. Over the generations, the variance in the population lowered and the optimization converged to a single ideal shape. The FO progress and results are given in Fig. 5. The decrease in shapes variance and increase of their performance may be seen in the figure left hand side. On the right hand side, we compared the resulting optimal shape, the default shape and a shape acquired using the less

\footnotetext{
${ }^{1}$ In the mesh size independence study, we compared the $\boldsymbol{f}_{\text {cost }}$ values obtained via HFDIB-based CFD to the results from a shape-conforming mesh. The mesh shown in Fig. 5-d) was used as a compromise between accuracy and computational costs.
} 
general methodology, where we did not stochastically add material, but we randomly moved 5 control points defining the diffuser shape. For details on the less general methodology, see [12].
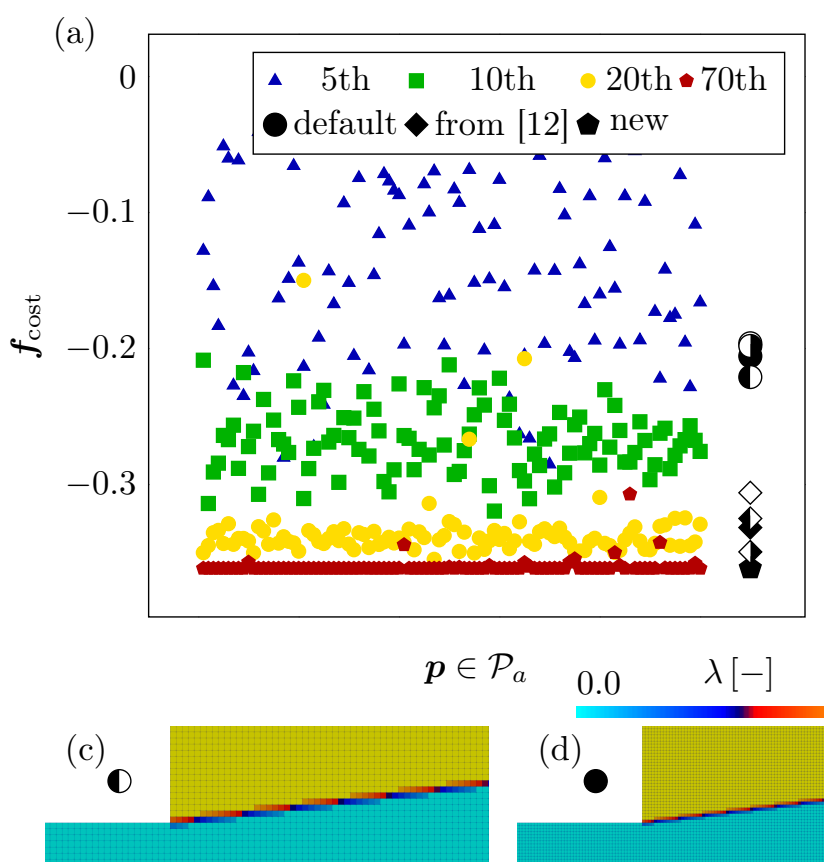

(b)

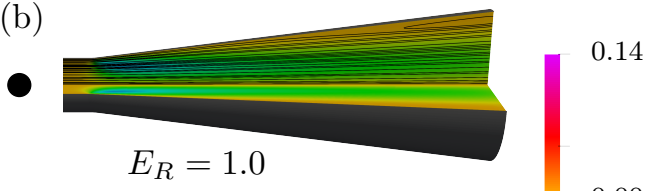

0.00

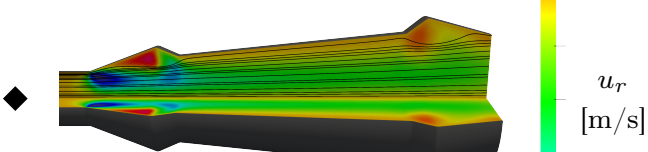

$r$

$E_{R}=1.61$

$\rightarrow x$

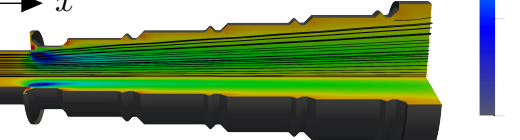

$E_{R}=1.76$

1.0
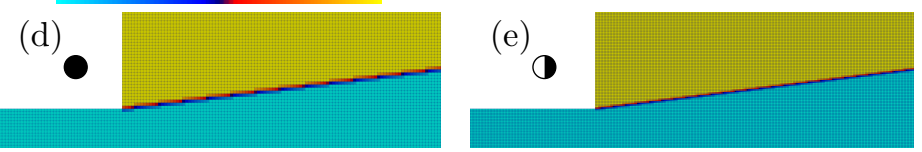

Figure 5: (a) Final optimization progress with generations indicated in the legend and the relative performance of the default shape (circle), shape found via the less general approach (square) and shape found via the proposed methodology (pentagon) highlighted. (b) Qualitative view of the three shapes highlighted in (a) colored by radial velocity and with indicated streamlines. Next, there is their relative energy efficiency $E_{R}=c_{p} / c_{p \text {,default. }}$ (c)-(e) Qualitative views of the three tested meshes colored by the $\lambda$ field. Used mesh corresponds to full symbols. Results from a shape-conforming mesh are marked by empty symbols.

The diffuser shape found by the newly proposed methodology had by $76 \%$ higher energy efficiency then the default diffuser shape and by $9 \%$ higher then the shape found via the less general approach. Note that altough the computing resources required to evaluate the optimization are quite high, MOEAs are highly scalable. In particular, each individuum of a single generation may be evaluated independently. Furthermore, the scalability of a single individuum evaluation is limited only by the scalability of the used CFD software. Consequently, the proposed methodology is able to effectively use the available computing resources. Furthermore, the parameter space sampling performed by MOEAs may be combined with machine learning to further improve the framework efficiency, see e.g. [15].

\section{Conclusion}

In the present contribution, we proposed a robust and general methodology for topology optimization in fluid dynamics that links MOEAs, CFD and HFDIB. The methodology was applied to increase the energy efficiency of a diffuser equipped as a part of an ejector. The diffuser shape found via the new methodology was $76 \%$ more energy-efficient than the default shape and roughly $9 \%$ more efficient than a shape found via an alternative shape optimization method. The main contribution of the new TO methodology lies in its robustness with respect to the cost function properties and in the fact that virtually no a-priory assumptions on the final optimized shape have to be made. However, to improve the methodology practical usability, it is necessary to extend it by a cost-efficient turbulence modeling, which boils down to implementing wall functions into the HFDIB method. 


\section{Acknowledgment}

The work was financially supported by the Czech Science Foundation (GA19-22173S), by the institutional support RVO:61388998, and by the Centre of Excellence for Nonlinear Dynamic Behaviour of Advanced Materials in Engineering CZ.02.1.01/0.0/0.0/15_003/0000493 (Excellent Research Teams) in the framework of Operational Programme Research, Development and Education.

\section{References}

[1] Rozvany, G.: Aims, scope, methods, history and unified terminology of computer-aided topology optimization in structural mechanics. structural and multidisciplinary optimization. Structural and Multidisciplinary Optimization. vol. 21: (04 2001). pp. 90-108.

[2] Sid, B., Domaszewski, M. \& Peyraut, F.: Topology optimization using an adaptive genetic algorithm and a new geometric representation. In Computer Aided Optimum Design in Engineering IX. WIT Press: (2005).

[3] Schoenauer, M.: Shape representations and evolution schemes. In Fifth Annual Conference on Evolutionary Programming. San Diego, United States: (1996).

[4] Jensen, J. \& Sigmund, O.: Topology optimization of photonic crystal structures: A highbandwidth low-loss t-junction waveguide. Journal of The Optical Society of America B-optical Physics. vol. 22: (06 2005). pp. 1191-1198.

[5] Ma, Z., Stalnov, O. \& Huang, X.: Design method for an acoustic cloak in flows by topology optimization. Acta Mechanica Sinica. vol. 35: (06 2019). pp. 964-971.

[6] Deb, K., Pratap, A., Agarwal, S. \& Meyarivan, T.: A fast and elitist multiobjective genetic algorithm: NSGA-II. IEEE Transs on Evol. Comput. vol. 6 no. 2: (2002). pp. 182-197.

[7] Hur, D., Lim, S., Izui, K. \& Nishiwaki, S.: Unified structural optimization method using topology optimization and genetic algorithms. Mechanical Engineering Journal. vol. 8: (06 2021).

[8] Boichot, R. \& Fan, Y.: A genetic algorithm for topology optimization of area-to-point heat conduction problem. International Journal of Thermal Sciences. vol. 108: (10 2016). pp. 209217.

[9] Koguchi, A., Yaji, K., Yamada, T., Izui, K. \& Nishiwaki, S.: Topology optimization method for unsteady state incompressible viscous flow based on a level set immersed boundary method. Transactions of the JSME (in Japanese). (04 2019).

[10] Kubo, S., Koguchi, A., Yaji, K., Yamada, T., Izui, K. \& Nishiwaki, S.: Level set-based topology optimization for two dimensional turbulent flow using an immersed boundary method. Journal of Computational Physics. vol. 446: (2021). page 110630.

[11] Šourek, M. \& Isoz, M.: Recent improvements in cfd solver for fully coupled particle-laden flows. In Proceedings of Topical Problems of Fluid Mechanics 2021: pp. 124-131. IT CAS: (02 2021).

[12] Kubíčková, L., Isoz, M. \& Haidl, J.: Increasing ejector efficiency via diffuser shape optimization. In Proceedings of Topical Problems of Fluid Mechanics 2021: pp. 79-86. IT CAS: (02 2021).

[13] OpenCFD. OpenFOAM: The Open Source CFD Toolbox. User Guide Version 1.4, OpenCFD Limited. Reading UK: (2007).

[14] Daniels, S., Rahat, A., Tabor, G., Fieldsend, J. \& Everson, R.: Automated shape optimization of a plane asymmetric diffuser using combined Computational Fluid Dynamic simulations and multi-objective Bayesian methodology. Int. J. Comp. Fl. Dyn. (2019). pp. 1683165-1$1683165-17$.

[15] Fan, Z., Hu, K., Li, F., Rong, Y., Li, W. \& Lin, H.: Multi-objective evolutionary algorithms embedded with machine learning - a survey. In 2016 IEEE Congress on Evolutionary Computation (CEC): pp. 1262-1266: (2016). 